\title{
TOPOLOGIES ON BOOLEAN ALGEBRAS DEFINED BY IDEALS AND DUAL IDEALS
}

\author{
by R. BEAZER \\ (Received 16 March, 1971)
}

Introduction. In the paper [5], Rema used the well-known fact that in a Boolean algebra $\mathscr{B}=\left\langle B ; \vee, \wedge,^{\prime} ; 0,1\right\rangle$ the binary operation $d: B \times B \rightarrow B$ defined by $d(a, b)=\left(a \wedge b^{\prime}\right) \vee\left(b \wedge a^{\prime}\right)$ is a "metric" operation to show that, if $D$ is any dual ideal of $\mathscr{B}$, then the sets $U_{p}=\{(x, y)$ : $d(x, y) \leqq p\}$, where $p \in D$, form a base for a uniformity of $\mathscr{B}$, the resulting topological space $\langle B ; T[D]\rangle$ being called an auto-topologized Boolean algebra. Recently, Kent and Atherton $[1,4]$ exhibited a family of topologies on an arbitrary lattice $\mathscr{L}$ defined in terms of ideals and dual ideals. More specifically, if $I$ and $D$ are respectively an ideal and a dual ideal of $\mathscr{L}$, then the $T[I: D]$ topology on $\mathscr{L}$ is the topology defined by taking the sets of the form $a^{*} \cap b^{+}$, where $a \in I, b \in D, a^{*}=\{x \in \mathscr{L}: x \geqq a\}$ and $b^{+}=\{x \in \mathscr{L}: x \leqq b\}$, as sub-base for the open sets. It is these topologies that are studied in this paper.

It is first shown that a $T[I: D]$ topology on a Boolean algebra $\mathscr{B}$ is an auto-topology if and only if $I$ is the "Boolean-complement" of $D$. The property of a topology on $\mathscr{B}$ being a $T[I: D]$ (auto-)topology is shown to be "productive" as well as being " $c$-hereditary" in that, if $S$ is a complete subalgebra of a Boolean algebra endowed with a $T[I: D]$ (auto-)topology, then the subspace topology on $S$ is a $T[I: D]$ (auto-)topology. Necessary and sufficient conditions are then established for a $T[I: D]$ topology to be Hausdorff and employed to show that a Hausdorff $T[I: D]$ topology is totally disconnected whereas an auto-topology is Hausdorff if and only if it is totally disconnected. Various connectedness properties of $T[I: D]$ topologies are studied in some detail and it is shown, in particular, that such a topology is connected if and only if $I$ is contained in the "lower section" of $D$ and that an auto-topology $T[D]$ is locally connected if and only if $D$ is a principal dual ideal. Finally, we show that a Boolean algebra admits a compact, Hausdorff $T[I: D]$ topology if and only if it is complete and atomic.

Notation and terminology. The topological concepts and results referred to throughout the paper can be found in [3], while the lattice-theoretic results are to be found in [2]. If $S$ is a nonempty subset of a Boolean algebra $\mathscr{B}$, then we denote the set $\left\{a^{\prime} ; a \in S\right\}$ by $S^{\prime}$ and refer to it as the Boolean complement of $S$. The usual partial ordering of $\mathscr{B}$ will be denoted by $\leqq$ and $[a, b]$ will denote the interval $\{x \in \mathscr{B}: a \leqq x \leqq b\}$. For the sake of brevity we frequently write $a . b$ instead of $a \wedge b$ for the lattice meet of $a$ and $b$ and $a \vee b$ for the lattice join. The symbols $\subseteq, \cup, \cap$ will be reserved for set inclusion, union and intersection respectively.

1. THEOREM 1.1. $T[I: D]$ is an auto-topology if and only if $D=I^{\prime}$ and, when this condition is satisfied, $T[I: D]=T[D]$.

Proof. Suppose that $T[I: D]$ coincides with the auto-topology $T[F]$ defined by the dual ideal $F$ of $\mathscr{B}$; then the set $\left\{U_{f}[a]: f \in F\right\}$, where $U_{f}[a]=\{x: d(x, a) \leqq f\}$, forms a base for 
the $T[F]$ neighbourhood system of the point $a \in \mathscr{B}$, and the set $\{[a \wedge q, a \vee p]: p \in D, q \in I\}$ forms a base for the $T[I: D]$ neighbourhood system of $a \in \mathscr{B}$. Now $d(x, a) \leqq f \leftrightarrow a \wedge f^{\prime} \leqq$ $x \leqq a \vee f$, so that $U_{f}[a]=\left[a \wedge f^{\prime}, a \vee f\right]$, and it follows that $\forall a \in \mathscr{B}, \forall p \in D, \forall q \in I, \exists f \in F$ such that $\left[a \wedge f^{\prime}, a \vee f\right] \subseteq[a \wedge q, a \vee p]$. On taking $a=0$, we deduce that every element in $D$ contains some element in $F$ and this implies that $D \subseteq F$. Furthermore, on taking $a=1$, it follows that $\forall q \in I, \exists f \in F$ such that $q \leqq f^{\prime}$, or equivalently $f \leqq q^{\prime}$, and so $q^{\prime} \in F$, which implies that $I \subseteq F^{\prime}$. Similarly $\forall a \in \mathscr{B}, \forall f \in F, \exists p \in D$ and $q \in I$ such that $[a \wedge q, a \vee p] \subseteq\left[a \wedge f^{\prime}, a \vee f\right]$. Taking $a=0$, we have that $\forall f \in F, \exists p \in D$ such that $p \leqq f$ and so $f \in D$, which shows that $F \subseteq D$. Again, taking $a=1$, we have that $\forall f \in F, \exists q \in I$ such that $f^{\prime} \leqq q$ and this implies that $F^{\prime} \subseteq I$. In summary then, $D=F$ and $I=F^{\prime}$; whence $D=I^{\prime}$.

The converse has been established by Atherton [1] who showed that, if this condition is satisfied, then $T[I: D]=T[D]$.

A property $\mathscr{P}$ of a topology on a Boolean algebra is said to be productive if and only if the product of any family of Boolean algebras, each being endowed with a topology possessing the property $\mathscr{P}$, also possesses $\mathscr{P}$.

THEOREM 1.2. The property of being a $T[I: D]$ topology is productive.

Proof. Suppose that $\left\{\left\langle\mathscr{B}_{\alpha} ; T\left[I_{\alpha}: D_{\alpha}\right]\right\rangle\right\}_{\alpha \in \Lambda}$ is an arbitrary family of Boolean algebras each endowed with a $T[I: D]$ topology. Let $D$ be the subset of the direct product $\mathscr{B}$ of the $\mathscr{B}_{\alpha}$ 's consisting of all functions $f \in \mathscr{B}$ with the property that $f(\alpha)=1_{\alpha}, \forall \alpha \in \Lambda$, except when $\alpha$ is in some finite subset $\left\{\alpha_{1}, \alpha_{2}, \ldots, \alpha_{n}\right\}$ of $\Lambda$, in which case $f\left(\alpha_{i}\right) \in D_{\alpha_{i}}$. Similarly, let $I$ be the subset of $\mathscr{B}$ consisting of all functions $f \in \mathscr{B}$ with the property that $f(\alpha)=0_{a}, \forall \alpha \in \Lambda$, except when $\alpha$ is in some finite subset $\left\{\alpha_{1}, \alpha_{2}, \ldots, \alpha_{n}\right\}$ of $\Lambda$, in which case $f\left(\alpha_{i}\right) \in I_{\alpha_{1}}$. Then it is easily shown that $D$ is a dual ideal and $I$ an ideal of $\mathscr{B}$, and we prove that the product topology $\prod_{\alpha \in \Lambda} T\left[I_{\alpha}: D_{\alpha}\right]$ on $\mathscr{B}$ coincides with the topology $T[I: D]$.

To this end, let $f \in U \in \prod_{\alpha \in \Lambda} T\left[I_{\alpha}: D_{\alpha}\right]$; then, by definition of the product topology, there exist open sets $U_{\alpha_{j}} \in T\left[I_{\alpha_{j}}: D_{\alpha_{j}}\right]\left(\alpha_{j} \in \Lambda, 1 \leqq j \leqq m\right)$ such that the corresponding sub-basic open sets $U_{\alpha_{j}}^{*}=\left\{f \in \mathscr{B}: f\left(\alpha_{j}\right) \in U_{\alpha_{j}}\right\}$ in $\prod_{\alpha \in \Lambda} T\left[I_{\alpha}: D_{\alpha}\right]$ satisfy $f \in \bigcap_{j=1}^{m} U_{\alpha_{j}}^{*} \subseteq U$. Now, since $f\left(\alpha_{j}\right) \in U_{\alpha_{j}}$ and $U_{\alpha_{j}} \in T\left[I_{\alpha_{j}}: D_{\alpha_{j}}\right]$, it follows that $\exists p_{\alpha_{j}} \in D_{\alpha_{j}}$ and $q_{\alpha_{j}} \in I_{\alpha_{j}}$ such that $\left[f\left(\alpha_{j}\right) \wedge p_{\alpha_{j}}, f\left(\alpha_{j}\right) \vee q_{\alpha_{j}}\right] \subseteq$ $U_{\alpha_{j}}(1 \leqq j \leqq n)$. Let $q \in \mathscr{B}$ be defined by $q(\alpha)=0_{\alpha}, \forall \alpha \in \Lambda$ except where $\alpha=\alpha_{j}$, when $q\left(\alpha_{j}\right)=q_{\alpha}(1 \leqq j \leqq m)$, and let $p \in \mathscr{B}$ be defined by $p(\alpha)=1_{\alpha}, \forall \alpha \in \Lambda$ except where $\alpha=\alpha_{j}$, when $p\left(\alpha_{j}\right)=p_{\alpha_{j}}(1 \leqq j \leqq m)$. Then $p \in D, q \in I$ and $[f \wedge q, f \vee p]$ is a $T[I: D]$-open neighbourhood of $f$ which is contained in $\bigcap_{j=1}^{m} U_{\alpha_{j}}^{*}$; for if $g \in[f \wedge q, f \vee p]$, then, in particular, $f\left(\alpha_{j}\right) \wedge q\left(\alpha_{j}\right) \leqq$ $g\left(\alpha_{j}\right) \leqq f\left(\alpha_{j}\right) \vee p\left(\alpha_{j}\right)$, so that $g\left(\alpha_{j}\right) \in\left[f\left(\alpha_{j}\right) \wedge q_{\alpha_{j}}, f\left(\alpha_{j}\right) \vee p_{\alpha_{j}}\right](1 \leqq j \leqq m)$, whence $g \in \bigcap_{j=1}^{m} U_{\alpha_{j}}^{*}$ Thus $\prod_{\alpha \in \Lambda} T\left[I_{\alpha}: D_{\alpha}\right] \subseteq T[I: D]$.

Conversely, suppose that $f \in U \in T[I: D]$; then $\exists p \in D, q \in I$ such that $f \in[f \wedge q, f \vee p] \subseteq U$. Now suppose that $p(\alpha)=1_{\alpha} \forall \alpha \in \Lambda-J, p\left(\alpha_{j}\right) \in D_{\alpha_{j}} \forall \alpha_{j} \in J$, where $J$ is a finite subset of $\Lambda$, and $q(\beta)=0_{\beta} \forall \beta \in \Lambda-K, q\left(\beta_{k}\right) \in I_{\beta_{k}} \forall \beta_{k} \in K$, where $K$ is a finite subset of $\Lambda$. Let $L=J \cup K$ and, for 
each $\gamma \in L$, consider the sub-basic $\prod_{\alpha \in \Lambda} T\left[I_{\alpha}: D_{\alpha}\right]$-open set $U_{\gamma}^{*}=\left\{b \in \mathscr{B}: b(\gamma) \in U_{\gamma}\right\}$, where $U_{\gamma}$ is the basic $T\left[I_{\gamma}: D_{\gamma}\right]$-open set $[f(\gamma) \wedge q(\gamma), f(\gamma) \vee p(\gamma)]$. Now $f \in \bigcap_{\gamma \in L} U_{\gamma}^{*} \subseteq[f \wedge q, f \vee p] \subseteq U$; for, if $g \in \bigcap_{\gamma \in L} U_{\gamma}^{*}$, then $g(\gamma) \in U_{\gamma}, \forall \gamma \in L$, or, equivalently, $f(\gamma) \wedge q(\gamma) \leqq g(\gamma) \leqq f(\gamma) \vee p(\gamma), \forall \gamma \in L$, and, if $\alpha \in \Lambda-L$, so that $\alpha \in \Lambda-J$ and $\alpha \in \Lambda-K$, then $q(\alpha)=0_{\alpha}$ and $p(\alpha)=1_{\alpha}$, which implies that $f(\alpha) \wedge q(\alpha) \leqq g(\alpha) \leqq f(\alpha) \vee p(\alpha), \forall \alpha \in \Lambda$, i.e., $g \in[f \wedge q, f \vee p]$. Hence $T[I: D] \subseteq \prod_{\alpha \in \Lambda} T\left[I_{\alpha}: D_{\alpha}\right]$ and therefore equality holds.

COROLLARY 1.3. The property of being an auto-topology is productive.

Proof. If each of the topologies $T\left[I_{\alpha}: D_{\alpha}\right]$ in the theorem is an auto-topology, then, by Theorem 1.1, $D_{\alpha}=I_{\alpha}^{\prime} \forall \alpha \in \Lambda$, and it is easily shown that the associated ideal $I$ and dual idea $D$ of $\mathscr{B}$ satisfy $D=I^{\prime}$. Hence the product topology on $\mathscr{B}$ is an auto-topology.

A property $\mathscr{P}$ of a topology on a Boolean algebra is said to be $c$-hereditary if and only if the subspace topology on any complete subalgebra of a Boolean algebra endowed with a topology possessing the property $\mathscr{P}$ also possesses $\mathscr{P}$.

THEOREM 1.4. The property of being a $T[I: D]$ topology is c-hereditary.

Proof. Let $S$ be a complete subalgebra of the Boolean algebra $\mathscr{B}$; for each $p \in D$, let $t_{p}=V\left(p^{+} \cap S\right)$ and form the dual ideal $D_{s}$ in $S$ generated by the set $T_{D}=\left\{t_{p}: p \in D\right\}$. Observe that, since $T_{D}$ is closed under finite meets, $D_{s}=\left\{s \in S: s \geqq t_{p}\right.$ for some $\left.p \in D\right\}$. For each $q \in I$, let $t_{q}=\wedge\left(q^{*} \cap S\right)$, form the ideal $I_{s}$ in $S$ generated by the set $T_{I}=\left\{t_{q}: q \in I\right\}$ and, once again, observe that $I_{s}=\left\{s \in S: s \leqq t_{q}\right.$ for some $\left.q \in I\right\}$. We show that the subspace topology $T[I: D] / S$ on $S$ is identical with $T\left[I_{s}: D_{s}\right]$. Let $a \in U \in T[I: D] / S$; then $\exists p \in D, q \in I$ such that $a \in[a \wedge q, a \vee p] \cap S \subseteq U$. Consider the interval $\left[a \wedge t_{q}, a \vee t_{p}\right]_{s}$ in $S$, i.e., $\left\{s \in S: a \wedge t_{q} \leqq s \leqq\right.$ $\left.a \vee t_{p}\right\}$; then, since $t_{p} \leqq p$ and $t_{q} \geqq q$, it follows that $\left[a \wedge t_{q}, a \vee t_{p}\right]_{s}=[a \wedge q, a \vee p] \cap S$. Hence $T[I: D] / S \subseteq T\left[I_{s}: D_{s}\right]$.

Conversely, let $a \in U \in T\left[I_{s}: D_{s}\right]$; then $\exists p_{1} \in D_{s}, q_{1} \in I_{s}$ such that $\left[a \wedge q_{1}, a \vee p_{1}\right]_{s} \subseteq U$. But $p_{1} \in D_{s} \leftrightarrow p_{1} \geqq t_{p}$ for some $p \in D$, and $q_{1} \in I_{s} \leftrightarrow q_{1} \leqq t_{q}$ for some $q \in I$. We show that $[a \wedge q, a \vee p] \cap S \subseteq\left[a \wedge q_{1}, a \vee p_{1}\right]_{s}$. Let $s \in[a \wedge q, a \vee p] \cap S$, so that $s \in S$ and $s \wedge a^{\prime} \leqq p$; then it follows that $s \wedge a^{\prime} \leqq t_{p} \leqq p_{1}$, or, equivalently, $s \leqq a \vee p_{1}$. Similarly, since $a^{\prime} \vee s \geqq q$ and $a^{\prime} \vee s \in S$, it follows that $a^{\prime} \vee s \geqq t_{q} \geqq q_{1}$, or, equivalently, $a \wedge q_{1} \leqq s$. Hence $s \in\left[a \wedge q_{1}, a \vee p_{1}\right]_{s}$ and therefore $T\left[I_{s}: D_{s}\right] \subseteq T[I: D] / S$.

\section{COROLLARY 1.5. The property of being an auto-topology is c-hereditary.}

Proof. If the topology $T[I: D]$ of the theorem is an auto-topology, then $D=I^{\prime}$ and it suffices to show that $D_{s}=I_{s}^{\prime}$. To this end let $s \in D_{s}$; then $\exists p \in D$ such that $s \geqq t_{p}=\mathrm{V}\left(p^{+} \cap S\right)$. Now, since $p=q^{\prime}$ for some $q \in I, t_{p}^{\prime}=\Lambda\left(p^{+} \cap S\right)^{\prime}=\Lambda\left(q^{*} \cap S\right)=t_{q}$ and so $s^{\prime} \leqq t_{q}$, which implies that $s^{\prime} \in I_{s}$, or, equivalently, $s \in I_{s}^{\prime}$. Hence $D_{s} \subseteq I_{s}^{\prime}$. Similarly, if $s \in I_{s}^{\prime}$, so that $s=r^{\prime}$ for some $r \in I_{s}$, then $\exists q \in I$ such that $t_{q}^{\prime} \leqq s$. Now $q=p^{\prime}$ for some $p \in D$, and so $t_{q}^{\prime}=\left[\wedge\left(q^{*} \cap S\right)\right]^{\prime}$ $=\mathrm{V}\left(q^{*} \cap S\right)^{\prime}=\mathrm{V}\left(p^{+} \cap S\right)=t_{p}$, which implies that $t_{p} \leqq s$ and therefore $s \in D_{s}$. Hence $I_{s}^{\prime} \subseteq D_{s}$, completing the proof. 
2. Connectedness properties. Prior to establishing necessary and sufficient conditions for $T[I: D]$ to be Hausdorff, we recall that an ideal (dual ideal) in the pseudo-complemented lattice $\mathscr{L}$ of all ideals (dual ideals) of a Boolean algebra $\mathscr{B}$ is said to be (algebraically) dense if and only if its pseudo-complement is the zero element of $\mathscr{L}$. We remark that an ideal $I$ of $\mathscr{B}$ is dense if and only if its upper section $I^{*}=\{x \in \mathscr{B}: x \geqq q, \forall q \in I\}$ contains only the element 1 , while a dual ideal $D$ is dense if and only if its lower section $D^{+}=\{x \in \mathscr{B}: x \leqq p, \forall p \in D\}$ contains only the element 0 .

THEOREM 2.1. The topology $T[I: D]$ is Hausdorff if and only if both I and $D$ are dense.

Proof. Suppose that $T[I: D]$ is Hausdorff and $x \in I^{*}$ but $x \neq 1$; then $\exists p_{1} \in D$ and $\exists q_{1}, q_{2} \in I$ such that $\left[q_{2}, 1\right] \cap\left[x \wedge q_{1}, x \vee p_{1}\right]=\emptyset$, which gives a contradiction on observing that the element $q=q_{1} \vee q_{2} \in I$ satisfies $q_{2} \leqq q$ and $x \wedge q_{1}=q_{1} \leqq q \leqq x \leqq x \vee p_{1}$ and therefore lies in the intersection. Hence $I^{*}=\{1\}$. Similarly, suppose that $x \in D^{+}$but $x \neq 0$; then $\exists p_{1}, p_{2} \in D$ and $\exists q_{1} \in I$ such that $\left[0, p_{2}\right] \cap\left[x \wedge q_{1}, x \vee p_{1}\right]=\emptyset$, which, on observing that $p=p_{1} \wedge p_{2} \in D$ satisfies $p \leqq p_{2}$ and $x \wedge q_{1} \leqq x \leqq p \leqq x \vee p_{1}$, gives a contradiction. Hence $D^{+}=\{0\}$.

Conversely, suppose that both $I$ and $D$ are dense, but $T[I: D]$ is not Hausdorff; then there exist distinct points $a, b \in \mathscr{B}$ such that every open neighbourhood of $a$ meets every open neighbourhood of $b$. Hence $[a \wedge q, a \vee p] \cap[b \wedge q, b \vee p] \neq \emptyset, \forall p \in D, \forall q \in I$. But $\exists x \in \mathscr{B}$ satisfying

$$
\begin{aligned}
x \in[a \wedge q, a \vee p] \cap[b \wedge q, b \vee p] & \leftrightarrow a q \vee b q \leqq x \leqq(a \vee p)(b \vee p) \\
& \leftrightarrow\left(a^{\prime} \vee b^{\prime}\right) p^{\prime} x \vee(a \vee b) q x^{\prime}=0 \\
& \leftrightarrow\left(a^{\prime} \vee b^{\prime}\right)(a \vee b) p^{\prime} q=0 \\
& \leftrightarrow d(a, b) p^{\prime} q=0
\end{aligned}
$$

and so it follows that $d(a, b) q \leqq p, \forall p \in D, \forall q \in I$. Whence

$$
\begin{aligned}
d(a, b) q \in D^{+}=\{0\}, \forall q \in I & \leftrightarrow q \leqq d^{\prime}(a, b), \forall q \in I \\
& \leftrightarrow d^{\prime}(a, b) \in I^{*}=\{1\} \\
& \leftrightarrow d(a, b)=0 \\
& \leftrightarrow a=b,
\end{aligned}
$$

giving a contradiction and therefore proving that $T[I: D]$ is Hausdorff.

COROLlary 2.2. An auto-topology $T[D]$ is Hausdorff if and only if $D$ is a dense dual ideal.

THEOREM 2.3. If $T[I: D]$ is Hausdorff, then it is totally disconnected.

Proof. It is, of course, well known that a Hausdorff, zero-dimensional space is totally disconnected and so, in proving the theorem, it suffices to show that each basic open set $[a \wedge q, a \vee p](p \in D, q \in I)$ is clopen. Now $x \in \mathrm{Cl}\left[a \wedge q_{1}, a \vee p_{1}\right]$, the closure of $\left[a \wedge q_{1}, a \vee p_{1}\right]$, 
if and only if every neighbourhood of $x$ meets $\left[a \wedge q_{1}, a \vee p_{1}\right]$ or, equivalently, $\mathscr{I}=$ $[x \wedge q, x \vee p] \cap\left[a \wedge q_{1}, a \vee p_{1}\right] \neq \emptyset, \forall p \in D, \forall q \in I$. But

$$
\begin{aligned}
\exists y \in \mathscr{I} & \leftrightarrow x q \vee a q_{1} \leqq y \leqq(x \vee p)\left(a \vee p_{1}\right) \\
& \leftrightarrow\left(x^{\prime} p^{\prime} \vee a^{\prime} p_{1}^{\prime}\right) y \vee\left(x q \vee a q_{1}\right) y^{\prime}=0 \\
& \leftrightarrow\left(x^{\prime} p^{\prime} \vee a^{\prime} p_{1}^{\prime}\right)\left(x q \vee a q_{1}\right)=0 \\
& \leftrightarrow a q_{1} x^{\prime} p^{\prime}=0 \text { and } a^{\prime} p_{1}^{\prime} x q=0 \\
& \leftrightarrow a x^{\prime} q_{1} \leqq p \text { and } q \leqq a \vee x^{\prime} \vee p_{1} .
\end{aligned}
$$

Hence $\mathscr{I} \neq \emptyset, \forall p \in D, \forall q \in I \leftrightarrow a x^{\prime} q_{1} \leqq p, \forall p \in D$ and $q \leqq a \vee x^{\prime} \vee p_{1}, \forall q \in I \leftrightarrow a x^{\prime} q_{1} \in D^{+}=\{0\}$ and $a \vee x^{\prime} \vee p_{1} \in I^{*}=\{1\} \leftrightarrow a x^{\prime} q_{1}=0$ and $a^{\prime} p_{1}^{\prime} x=0 \leftrightarrow a \wedge q_{1} \leqq x \leqq a \vee p_{1}$. It follows now that $\left[a \wedge q_{1}, a \vee p_{1}\right]$ is clopen and the theorem is proved.

COROLLARY 2.4. An auto-topology is Hausdorff if and only if it is totally disconnected.

Proof. It is well known that $\mathrm{cmp}(a)$, the component of $a$, is contained in the intersection of all clopen sets containing the point $a$ and so, since the $T[D]$-open sets $[0, p](p \in D)$ are clopen, it follows that $\operatorname{cmp}(0) \subseteq \bigcap_{p \in D}[0, p]=D^{+}$. We show that the subspace $D^{+}$is indiscrete and therefore connected. To this end, let $V$ be an open set containing the element $l$ in the subspace $D^{+}$, so that $V=U \cap D^{+}$for some $T[D]$-open set $U$ containing $l$. Then $\exists p \in D$ such that $\left[l \wedge p^{\prime}, l \vee p\right] \cap D^{+} \subseteq V$. Furthermore, $D^{+} \subseteq\left[l \wedge p^{\prime}, l \vee p\right]$; for, if $x \in D^{+}$, so that $x \leqq p, \forall p \in D$, then $d(x, l) \leqq x \vee l \leqq p$ and so $x \in\left[l \wedge p^{\prime}, l \vee p\right]$. It follows now that $D^{+}=V$ and so the only open sets in the subspace $D^{+}$are itself and the empty set. Hence $D^{+}$is an indiscrete subspace. Now $\mathrm{cmp}(0)$ is the largest connected set containing the element 0 and so, by the connectedness of $D^{+}, \operatorname{cmp}(0)=D^{+}$. Hence, if $\langle B ; T[D]\rangle$ is totally disconnected, $D^{+}=\operatorname{cmp}(0)=\{0\}$ and it follows, by Corollary 2.2 , that $\langle B ; T[D]\rangle$ is Hausdorff.

THEOREM 2.5. The topology $T[I: D]$ is connected if and only if $I \subseteq D^{+}$.

Proof. Suppose that $T[I: D]$ is connected. Let $I_{m}$ be an arbitrary maximal ideal in $\mathscr{B}$ and let $p \in D, q \in I$ be given; then the set $\left\{[a \wedge q, a \vee p]: a \in I_{m}\right\}$ forms an open cover of $I_{m}$ and, by a well-known property of maximal ideals, the set $\left\{[b \wedge q, b \vee p]: b \in I_{m}^{\prime}\right\}$ forms an open cover of $\mathscr{B}-I_{m}$. Hence the open sets of $U=\bigcup_{a \in I_{m}}[a \wedge q, a \vee p], V=\bigcup_{b \in I_{m^{\prime}}}[b \wedge q, b \vee p] \operatorname{cover} \mathscr{B}$ and therefore cannot be disjoint. This implies that $\exists a, c \in I_{m}$ such that $[a \wedge q, a \vee p] \cap\left[c^{\prime} \wedge q, c^{\prime} \vee p\right] \neq$ $\emptyset \leftrightarrow \exists x \in \mathscr{B}$ such that

$$
\begin{aligned}
\left(a \vee c^{\prime}\right) q \leqq x \leqq a c^{\prime} \vee p & \leftrightarrow\left(a^{\prime} \vee c\right) p^{\prime} x \vee\left(a \vee c^{\prime}\right) q x^{\prime}=0 \\
& \leftrightarrow p^{\prime} q\left(a^{\prime} \vee c\right)\left(a \vee c^{\prime}\right)=0 \\
& \leftrightarrow q p^{\prime} d\left(a, c^{\prime}\right)=0 \leftrightarrow q p^{\prime} \leqq d(a, c) \in I_{m} .
\end{aligned}
$$

Hence $q p^{\prime} \in I_{m}$, so that, since $I_{m}$ is an arbitrary maximal ideal and the intersection of all maximal ideals of $\mathscr{B}$ contains only the element 0 , it follows that $q \leqq p, \forall p \in D, \forall q \in I$. Therefore $I \subseteq D^{+}$.

Conversely, suppose that $I \subseteq D^{+}$and let $C$ be any clopen subset of $\langle B ; T[I: D]\rangle$. Then either $C=\emptyset$ or $\exists a \in C$. In the latter case suppose that $\exists b \in \mathscr{B}-C$. Then $\exists p_{1} \in D, q_{1} \in I$ such 
that $\left[b \wedge q_{1}, b \vee p_{1}\right] \subseteq \mathscr{B}-C$. Also, since $C$ is open, $\exists p_{2} \in D, q_{2} \in I$ such that $\left[a \wedge q_{2}, a \vee p_{2}\right] \subseteq C$ and so these intervals are disjoint. But $I \subseteq D^{+} \leftrightarrow q \leqq p, \forall p \in D, \forall q \in I$ and we observe that the element $s=b q_{1} \vee a q_{2}$ lies in their intersection, giving a contradiction. Therefore, if $C$ is clopen, then either $C=\emptyset$ or $C=\mathscr{B}$; whence the space is connected.

THEOREM 2.6. An auto-topology $T[D]$ is locally connected if and only if $D$ is a principal dual ideal.

Proof. Suppose that $T[D]$ is a locally connected auto-topology on the Boolean algebra $\mathscr{B}$. Then there exists a base $\sigma$ for $T[D]$ consisting of connected open sets. Let $U$ be any member of $\sigma$ containing the least element of $\mathscr{B}$. Then $\exists p \in D$ such that $p^{+} \subseteq U$, which, since $p^{+}$is a nonempty clopen set and therefore clopen in the subspace $U$ of $T[D]$, implies that $p^{+}=U$. Now suppose that $D$ is non-principal. Then $\exists p_{1} \in D$ such that $p_{1}<p$ and so the clopen set $p_{1}^{+}$is properly contained in the connected set $p^{+}$, giving a contradiction. Hence $D$ is a principal dual ideal of $\mathscr{B}$.

Conversely, suppose that $T[D]$ is induced by the principal dual ideal $D=p^{*}$ generated by $p$. Then it is is obvious that the set $\left\{\left[a \wedge p^{\prime}, a \vee p\right]: a \in \mathscr{B}\right\}$ forms a base for $T[D]$. Furthermore these intervals are connected sets; for otherwise there exists a nonempty clopen set $U_{a}$, containing the element $a$, in the subspace $\left[a \wedge p^{\prime}, a \vee p\right]$ and distinct from it, which, since $U_{a}$ must be $T[D]$-open, implies that $\left[a \wedge p^{\prime}, a \vee p\right] \subseteq U_{a}$. It follows that $T[D]$ is locally connected.

The preceding theorem characterizes principal dual ideals of a Boolean algebra $\mathscr{B}$ in terms of a property of the associated auto-topologies, while Corollary 2.2. may be regarded as a characterization of dense dual ideals of $\mathscr{B}$. The following theorem characterizes, in the same way, the maximal dual ideals of $\mathscr{B}$.

THEOREM 2.7. If $T[D]$ is an auto-topology on $\mathscr{B}$, induced by the dual ideal $D$, then $D$ is maximal if and only if $\langle B ; T[D]\rangle$ is non-discrete and, for all $a \in \mathscr{B}$, either $a^{*}$ or $a^{+}$is an open set.

Proof. If $D$ is a maximal dual ideal of $\mathscr{B}$, then it is proper, so that $T[D]$ is non-discrete; and, furthermore, if $a \in \mathscr{B}$, then either $a \in D$ or $a^{\prime} \in D$. In the first case the set $U_{a}[a]=a^{+}$is open, while in the second the set $U_{a} \cdot[a]=a^{*}$ is open.

Conversely, suppose that $T[D]$ is a non-discrete auto-topology on $\mathscr{B}$ with the property that, for all $a \in \mathscr{B}$, either $a^{+}$or $a^{*}$ is open. Then $D$ is proper and, furthermore, if $a^{+}$is open, $\exists p \in D$ such that $U_{p}[a]=\left[a \wedge p^{\prime}, a \vee p\right] \subseteq a^{+}$, which implies that $a \vee p \leqq a$, or, equivalently, $p \leqq a$, and so $a \in D$. In the event that $a^{*}$ is open, $\exists p \in D$ such that $U_{p}[a]=\left[a \wedge p^{\prime}, a \vee p\right] \subseteq a^{*}$, which implies that $a \wedge p^{\prime} \geqq a$, or, equivalently, $p \leqq a^{\prime}$, and so $a^{\prime} \in D$. Hence $D$ is a proper dual ideal possessing the property that, for all $a \in \mathscr{B}$, either $a \in D$ or $a^{\prime} \in D$ and $D$ is therefore maximal.

\section{Compact Hausdorff $T[I: D]$ topologies.}

THEOREM 3.1. If a Boolean algebra admits a compact, Hausdorf $T[I: D]$ topology, then it is complete.

Proof. Let $X$ be any nonempty subset of a Boolean algebra $\mathscr{B}$ admitting a compact, 
Hausdorff $T[I: D]$ topology and let $\mathscr{L}_{X}$ be the set of all lower bounds of $X$; then ${ }^{\circ} \mathscr{L}_{X}$ is an ideal of $\mathscr{B}$ and consequently the identity map $n: \mathscr{L}_{X} \rightarrow \mathscr{L}_{X}$ is a net in $\mathscr{L}_{X}$ which, since $\langle B ; T[I: D]\rangle$ is compact, has a cluster point $c$. Let $p \in D, q \in I$ be given; then, since $n$ is frequently in the open neighbourhood [ $c \wedge q, c \vee p$ ], it follows that $\forall a \in \mathscr{L}_{X}, \exists b \in \mathscr{L}_{X}$ such that $b \geqq a$ and $b \in[c \wedge q, c \vee p]$. Whence $c \wedge q<b \leqq c \vee p$, from which it follows that $c \wedge q \in \mathscr{L}_{X}$ and $a \leqq c \vee p, \forall a \in \mathscr{L}_{X}$. Hence, since $p$ and $q$ were arbitrarily chosen, it follows that

$$
c \wedge q \leqq x, \forall x \in X, \forall q \in I \leftrightarrow q \leqq c^{\prime} \vee x, \forall x \in X, \forall q \in I \leftrightarrow c^{\prime} \vee x \in I^{*}, \forall x \in \mathscr{L}_{X} .
$$

Also

$$
a \leqq c \vee p, \forall a \in \mathscr{L}_{X}, \forall p \in D \leftrightarrow a \wedge c^{\prime} \leqq p, \forall a \in \mathscr{L}_{X}, \forall p \in D \leftrightarrow a \wedge c^{\prime} \in D^{+} .
$$

But $T[I: D]$ is Hausdorff, or, equivalently, $I^{+}=\{1\}, D^{*}=\{0\}$, and so $c^{\prime} \vee x=1, \forall x \in X$, and $a \wedge c^{\prime}=0, \forall a \in \mathscr{L}_{X}$, i.e., $c \in \mathscr{L}_{X}$ and $a \leqq c, \forall a \in \mathscr{L}_{X}$, so that $c$ is the greatest lower bound on the set $X$. It follows that $\mathscr{B}$ is complete.

THEOREM 3.2. A Boolean algebra admits a compact, Hausdorff $T[I: D]$ topology if and only if it is complete and atomic.

Proof. Let $\mathscr{B}$ be a Boolean algebra and suppose that $\langle B ; T[I: D]\rangle$ is compact and Hausdorff. Then, by the preceding theorem, $\mathscr{B}$ is complete and it remains only to show that $\mathscr{B}$ is atomic. To this end, let $p$ be an arbitrary element in the dual ideal $D$ distinct from the element 1. Then $\exists q \in I$ such that $q \geqq p$; otherwise $p \geqq q, \forall q \in I$, so that $p \in I^{*}=\{1\}$, whence $p=1$. Let $I_{p}$ be any prime ideal of $\mathscr{B}$ such that $p \in I_{p}$ but $q \notin I_{p}$, the existence of such an ideal being well known. Now

$$
\mathscr{C}=\left\{[a \wedge q, a \vee p],[b \wedge q, b \vee p]: a \in I_{p}, b \in \mathscr{B}-I_{p}\right\}
$$

is an open cover of $\mathscr{B}$ and so, since $T[I: D]$ is compact, $\exists$ a finite sub-cover

$$
\mathscr{C}^{*}=\left\{\left[a_{i} \wedge q, a_{i} \vee p\right],\left[b_{j} \wedge q, b_{j} \vee p\right]: 1 \leqq i \leqq m, 1 \leqq j \leqq n\right\}
$$

of $\mathscr{B}$. We assert that $\mathscr{C}^{* *}=\left\{\left[a_{i} \wedge q, a_{i} \vee p\right]: 1 \leqq i \leqq m\right\}$ is an open cover of $I_{p}$; for, if not, $\exists a \in I_{p}$ such that $a \in\left[b_{j} \wedge q, b_{j} \vee p\right]$ for some $j$. But $a \in I_{p}$ and $b_{j} \wedge q \leqq a$ implies that $b_{j} \wedge q \in I_{p}$, which, since $I_{p}$ is prime, implies that either $b_{j} \in I_{p}$ or $q \in I_{p}$, both of which give a contradiction. Hence $I_{p} \subseteq \bigcup_{i=1}^{n}\left[a_{i} \wedge q, a_{i} \vee p\right]$ so that $x \in I_{p} \rightarrow x \leqq \bigvee_{i=1}^{n}\left(a_{i} \vee p\right)=p \vee \bigvee_{i=1}^{n} a_{i} \in I_{p}$. Therefore $I_{p}$ is a principal ideal of $\mathscr{B}$ generated by $m$, say. But an ideal in $\mathscr{B}$ is prime if and only if it is maximal and so it follows that $m$ is a maximal element in $\mathscr{B}$. Furthermore, since the complement of a maximal element in $\mathscr{B}$ is an atom, we have shown that $\forall p \in D(p \neq 1), \exists$ an atom $a \leqq p^{\prime}$.

Let $a_{p}$ be the join of all atoms contained in $p^{\prime}$, which exists since $\mathscr{B}$ is complete; we show that $p^{\prime}=a_{p}$. For, if $p^{\prime}>a_{p}>0$, let $x$ be the relative complement of $a_{p}$ in the Boolean interval $\left[0, p^{\prime}\right]$, so that $0<x<p^{\prime}$ and $a_{p} \wedge x=0$. Then $p<x^{\prime}$, which implies that $x^{\prime} \in D$ and $x^{\prime} \neq 1$. Therefore $\exists$ an atom $b \leqq x^{\prime \prime}=x$, whence $b<p^{\prime}$, so that $b$ is an atom contained in $p^{\prime}$, which implies that $b \leqq a_{p}$. Then $0<b \leqq a_{p} \wedge x=0$, so that $b=0$, giving a contradiction. Hence $\forall p \in D, p^{\prime}$ is the join of all atoms it contains. Now we show that every element of $\mathscr{B}$ contains 
an atom. Since $T[I: D]$ is Hausdorff, $D^{+}=\{0\}$ and therefore, since $\bigwedge_{p \in D} p \in D^{+}$, it follows that $\bigwedge_{p \in D} p=0$, which implies that $\bigvee_{p \in D} p^{\prime}=1$. Each $p^{\prime}$ is, as we have shown, a join of atoms of $\mathscr{B}$ and therefore the element 1 is the join of all atoms of $\mathscr{B}$. Let $\mathscr{A}$ be the set of all atoms of $\mathscr{B}$ and suppose that some element $x \in \mathscr{B}$ contains no member of $\mathscr{A}$. Then $a \wedge x=0, \forall a \in \mathscr{A}$ and so $0=\bigvee_{a \in \mathscr{A}}(a \wedge x)=x \wedge \bigvee_{a \in \mathscr{A}} a=x \wedge 1=x$. Therefore every nonzero element of $\mathscr{B}$ contains an atom and so $\mathscr{B}$ is atomic.

Conversely, if $\mathscr{B}$ is complete and atomic, or, equivalently, $\mathscr{B}=2^{N}$ for some cardinal $N$, then, since each two-element Boolean algebra endowed with the discrete topology is a $T[I: D]$ topologized Boolean algebra and the property of being such a topology is productive, it follows that $\mathscr{B}$ admits a compact, Hausdorff $T[I: D]$ topology.

\section{REFERENCES}

1. C. R. Atherton, Concerning intrinsic topologies on Boolean algebras and certain bicompactly generated lattices, Glasgow Math. J. 11 (1970), 156-161.

2. G. Birkhoff, Lattice theory, 3rd edition, Amer. Math. Soc. Colloquium Publications 25 (Providence, R.I., 1967).

3. J. L. Kelley, General topology (Princeton, 1955),

4. D. C. Kent and C. R. Atherton, The order topology in a bicompactly generated lattice, $J$. Australian Math. Soc. 8 (1968), 345-349.

5. P. S. Rema, Auto-topologies in Boolean algebras, J. Indian Math. Soc. 30 (4) (1966), 221-243.

GLASGOW, G12 8QQ 